\title{
A Novel Technique for Fiber-Matrix Bond Strength Determination for Rupturing Fibers
}

\author{
Karthikeyan H. Obla \& Victor C. Li \\ Advanced Civil Engineering Materials Research Laboratory, University of Michigan, Ann Arbor, MI 48109. \\ 2125, USA
}

(Received 28 April 1994; accepted 17 February 1995)

\section{Abstract}

In this paper a novel technique for determination of fiber-cement bond strength has been presented. The technique involves imaging and measurement of the protruding fiber length from a tensile fracture surface of the composite. The bond property is then inferred from this measured averaged protruding length. The analytic tool for such interpretations accounts for fiber orientation and length distributions. This technique can be applied to any composite system involving fibers that rupture after the matrix cracks under tensile loading. Composite tensile strengths have been predicted using the inferred bond strength and an analytic model developed elsewhere. Good correlations have been obtained between predictions and the experimental observations thus indicating that the bond strength determination technique is indeed accurate.

Keywords: Fiber, matrix, bond strength, carbon fibers, composite, critical fiber length, protruding, tensile strength, length variation, orientation, diameter, fracture surface, embedment length.

\section{INTRODUCTION}

Fiber-matrix bond strength is an important property that controls mechanical properties of the composites. With fine and brittle fibers like carbon fibers (6-20 $\mu \mathrm{m}$ in diameter), it is difficult to conduct a direct pullout test to obtain bond properties. The problem is actually twofold: (i) it is extremely difficult to cast a specimen with the fiber embedded straight in the matrix and maintain alignment without breaking the fiber; (ii) it is very difficult to load the fiber in a pull-out test without breaking the fiber at the grip where stress concentration occurs. Still some attempts have been made to characterize the bond strength of carbon fibers in cement by the direct pullout test. ${ }^{1,2}$ These attempts till now have been met with marginal success judging from the wide variation in the measured bond strengths.

In this paper, a novel technique for fibermatrix bond strength determination is presented. This technique makes use of the measured average length of protruding fibers from the composite tensile specimen fracture surface and by virtue of an analytic expression relates this length to the bond strength. This alternative technique avoids some of the difficulties involved in single fiber pullout specimen preparation and testing. It is also an in-situ test, in the sense that average bond property is measured for the interfaces in the composite of actual fiber volume fraction, rather than in a specimen with artificially low fiber volume fraction when a single fiber is used. Using this technique the fiber-cement bond strength of different types of carbon fibers has been estimated. The carbon fiber-cement composite tensile strength has been predicted using these values of bond strengths. ${ }^{3}$ The predictions seem to agree well with experimentally measured uniaxial composite tensile strengths thus indicating that the bond strength estimates are accurate. With the successful development of this technique it is hoped that in the future carbon fiber cement composites can be designed successfully to demonstrate optimum properties. Though in this paper the emphasis is given on investigating 
the bond strength of carbon fibers in cement, the developed technique can be applied for any brittle matrix reinforced with fibers that rupture after the matrix cracks.

\section{THEORETICAL BACKGROUND}

If a cement composite reinforced with short random carbon fibers is subjected to tension, the cement matrix would crack first before the carbon fibers rupture due to its low tensile strain capacity $(0.01 \%$ for cement vs $1-2.5 \%$ for carbon fibers). Once the crack has occurred and the composite is subjected to further loading, the fibers would start debonding. For short embedment length or low bond strength fiber pullout can occur. Otherwise, fiber rupture can happen during the course of debonding if the fiber tensile stress reaches its failure strength.

After the two fracture surfaces have been completely separated (after all the fibers have completely pulled out with some broken) the fibers protruding from the fracture surface would have different lengths. Fibers that rupture would have a zero protruding length. This is because the maximum fiber stress occurs at the crack and the fibers will break there (which would be the case if the fiber diameter and strength do not vary along its length and there is no fiber bending rupture). However, all fibers with embedment lengths less than $L_{c}$, where

$$
L_{\mathrm{c}}=\frac{d_{\mathrm{f}} \sigma_{\mathrm{fu}}}{4 \tau}
$$

would pull out. ${ }^{4}$ In eqn (1), $\tau, d_{\mathrm{f}}, \sigma_{\mathrm{f}}$ and $\sigma_{\mathrm{fu}}$ are the fiber/matrix bond strength, fiber diameter and strength, respectively.

A composite reinforced with continuous aligned fibers would result in a fracture surface having zero fiber protruding length as all the fibers have an embedment length larger than $L_{c}$ and hence would have ruptured at the crack itself. However, in a composite reinforced with short random fibers, the protruding lengths would vary between 0 and $L_{\mathrm{c}}$. By assuming that the embedment lengths follow a uniform distribution and knowing the fiber length, it would be possible to predict the average length of fiber protruding from the specimen fracture surface for a given value of bond strength. Conversely, if the averaged protruding fiber length from a fracture surface is measured, then the bond strength can be estimated. This is the basic philosophy of this technique of bond strength determination. There are some additional factors that have to be taken into account. They are:

(1) Inclined fibers offer higher bridging stress and so would have a shorter protruding length.

(2) Carbon fibers have a fiber length variation.

(3) The measured fiber protruding length is based on a 2-D projected image. The actual fiber length would hence be longer.

These factors are explained in detail below.

(1) Inclined fiber effect on bridging force: It has been observed ${ }^{5}$ that polymeric fibers inclined to the crack surface offer higher bridging force than fibers perpendicular to the crack face. This is analogous to a flexible rope passing over a friction pulley. The increase in bridging force is provided by a factor $\mathrm{e}^{f \phi}$ where $f$ is the snubbing coefficient that takes the value between 0 and 1 and $\phi$ is the angle of inclination between the fiber and matrix crack face normal varying between 0 and $\pi / 2$. Thus it can be explained that a fiber with inclined angle $\phi$ would rupture if its embedment length is greater than $L_{\mathrm{c}} \mathrm{e}^{-f \phi}$. Application of this concept to brittle carbon fiber is at best a crude approximation, since carbon fibers are known to fail by bending rupture. The present model should be more accurate for carbon fibers with higher tensile strain.

(2) Fiber length variation: Carbon fibers have been found to display a fiber length variation. ${ }^{6,7}$ This variation could be due to the fiber manufacturing technique (particularly true for pitch type fiber). Fibers could also break when they are mixed with other constituents in a mixer. The length variation of the fibers after mixing has been measured. This technique has been explained previously in detail. The fiber length distribution after mixing has been approximated by a probability density function (the fiber length varies between $L_{1}$ and $L_{2}$ ) and this effect is included in relating the experimentally measured average fiber protruding length to the fiber/matrix bond strength.

(3) 2-D projection: The fiber length protruding from the tensile specimen fracture surface is measured by an Environmental Scanning Elec- 


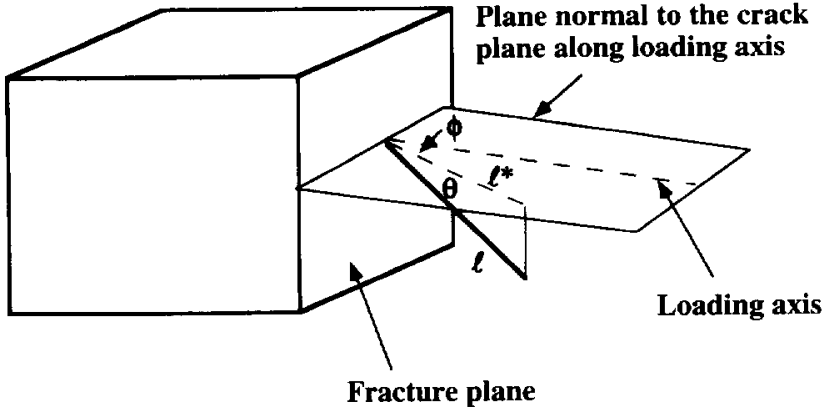

Fig. 1. Two dimensional image of the fiber protruding from the fracture surface as perceived by the eye.

tron Microscope (ESEM). This is discussed in more detail in Section 3. With this technique only a 2-D view of the protruding fiber is obtained and the fiber length measured will certainly be less than the actual fiber protruding length which would have a 3-D orientation. The actual protruding fiber length can be interpreted from this 2-D picture by assuming that the protruding fiber has a perfectly uniform spatial orientation. In Fig. $1, l$ is the actual protruding length of the fiber and $l^{*}$ is the fiber length measured by the ESEM which is a 2-D picture. If the angle made by the fiber with the plane normal to the crack plane along the loading axis (different from $\phi$ ) is $\theta$ then it can be easily seen that $l^{*}=l \cos \theta$ and a uniform 3-D distribution of the fiber in the composite implies the probability density distributions of:

$$
\begin{array}{ll}
p(\phi)=\sin \phi & 0 \leq \phi \leq \pi / 2 \\
p(\theta)=1 /(2 \phi) & -\phi \leq 0 \leq \phi .
\end{array}
$$

Thus to interpret the actual protruding fiber length from a 2-D picture of the protruding length these probability density functions for protruding length are used.

Akihama et $a l^{8}$ have measured the average protruding length of the fibers along the tensile specimen fracture surface. They equated the average protruding length to $L_{\mathrm{c}} / 2$ and thus computed the bond strength values. Based on the arguments above it is noted that their calculated $\tau$ value would be correct only if the fibers are aligned perpendicular to the crack face (along the loading axis) and are all of uniform length $L_{\mathrm{c}}$. The actual carbon fiber has a variable length distribution and has random orientation and hence the calculated $\tau$ values would be incorrect.

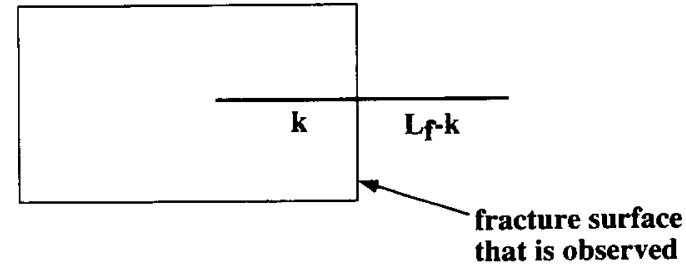

Fig. 2. Fiber protruding out of the fracture surface along the loading axis with embedment length $k$ varying between 0 and $L_{\mathrm{f}}$.

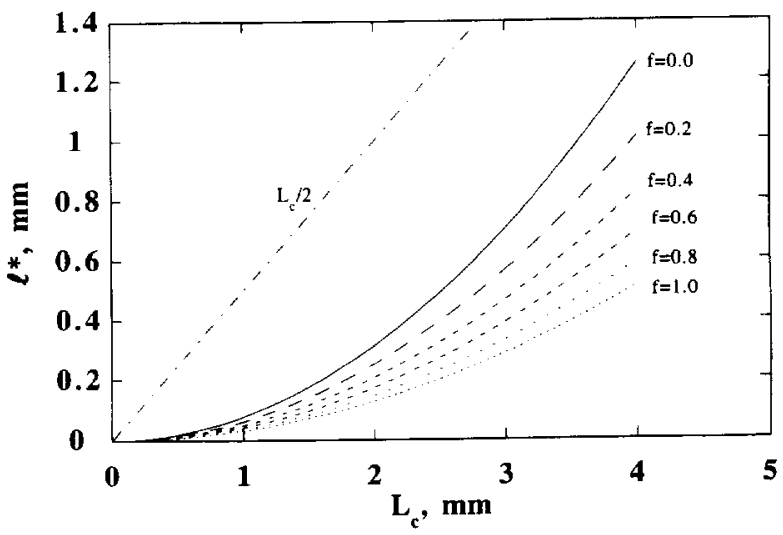

Fig. 3. Generic bond strength determination chart for fibers that have a constant length.

\subsection{Development of $l^{*}$ vs $L_{\mathrm{c}}$ relation}

(a) For constant $L_{f}$

If all the fibers are of length $L_{2}$ where $L_{2}>2 L_{\mathrm{c}}$ then the average fiber length protruding out of the fracture surface would be:

$$
\begin{aligned}
l^{*}= & 2 \int_{0}^{\pi / 2} \int_{-\phi}^{\phi} \int_{L_{1:}}^{L_{2}} p(k) p(\theta) p(\phi) \\
& \times\left(L_{2}-k\right) \cos \theta \mathrm{d} k \mathrm{~d} \theta \mathrm{d} \phi
\end{aligned}
$$

where $L_{\mathrm{f} 2}=L_{2}-L_{\mathrm{c}} \mathrm{e}^{-f \phi}$.

The above integral can be computed numerically. From Figs 1 and 2, for a fiber of protruding length $\left(l=L_{2}-k\right)$ the $2-\mathrm{D}$ measured length is $\left(L_{2}-k\right) \cos \theta$. Since the embedment length $k$ can vary uniformly between 0 and $L_{2}$, $p(k)=1 / L_{2} \cdot p(\phi)$ and $p(\theta)$ are given by eqns $(2)$ and (3), respectively. The lower limit of $k$ is obtained by making sure that the contribution to $l^{*}$ is obtained only from those fibers that pull out, i.e. $L_{\mathrm{f}}-k<L_{\mathrm{c}} \mathrm{e}^{-f \phi}$. Figure 3 shows the plot between $l^{*}$ and $L_{\mathrm{c}}$ generated from eqn (4) for different values of $f$ with $L_{2}=8.1 \mathrm{~mm}$. It can be seen that if $\tau$ was decreased, $L_{\mathrm{c}}$ would increase and since there would be less fiber breakage, $l^{*}$ should increase. For a given value of $f$, the 
$l^{*}-L_{\mathrm{c}}$ relation shown in Fig. 3 is consistent with the above physical concept. If $f$ is increased, then for the same $\tau$ value there would be more fiber breakage and so $l^{*}$ should decrease. Figure 3 captures this idea also.

\section{(b) For variable $L_{f}$ distribution}

The physical explanation of the development of the relation between $l^{*}$ vs $L_{c}$ is brought out here and the detailed governing equations are summarized in the appendix. It is assumed that $L_{1} \leq 2 L_{\mathrm{r}} \leq 2 L_{\mathrm{c}} \leq L_{2}$ (where $L_{\mathrm{r}}=L_{\mathrm{c}} \mathrm{e}^{-f \pi / 2}$ ). This implies that short fibers $\left(L_{\mathrm{f}}\right.$ close to $\left.L_{1}\right)$ can pull out, whereas some long fibers $\left(L_{\mathrm{f}}\right.$ close to $\left.L_{2}\right)$ can rupture. If the inequalities are not observed, then the bond strength predictions will not be valid. However, it is observed that carbon fibers typically satisfy these assumptions. Bending rupture of the fiber is assumed to be absent. The fibers are assumed to have a uniform strength and diameter. Fibers are assumed to have a uniform 3-D random distribution without clumping in the matrix. The appropriateness of these simplifying assumptions will depend on the specific fiber type.

The whole fiber length range is divided into three sectors and the average protruding length is calculated for each sector.

$L_{1} \leq L_{\mathrm{f}} \leq 2 L_{\mathrm{r}}$ : In this sector there cannot be any fiber rupture. Figure 2 shows a single fiber, oriented along the loading axis, embedded in the matrix. Since $k$ is the length of the fiber inside the matrix, the 2-D view of the fiber that has pulled out (if the fiber had broken then there should be zero protruding length) should have a length of $\left(L_{\mathrm{f}}-k\right) \cos \theta$. The average protruding length of fiber for this sector would be $l_{i}^{*}$ given in the appendix.

$2 L_{\mathrm{r}}<L_{\mathrm{f}} \leq 2 L_{\mathrm{c}}$ : In this sector there could be fiber rupture. Fibers with $L_{\mathrm{f}}<2 L_{\mathrm{d}}$ (where $L_{\mathrm{d}}=L_{\mathrm{c}} \mathrm{e}^{-f \phi}$ ) can never rupture and so the average protruding length would be $\left(L_{\mathrm{f}}-k\right) \cos \theta$. Fibers of $L_{\mathrm{f}}>2 L_{\mathrm{d}}$ can rupture if the embedment length $l>L_{\mathrm{d}}$. For those fibers with $l>L_{\mathrm{d}}$ the protruding length is taken as zero and for those with $l<L_{\mathrm{d}}$ the protruding length is taken as $\left(L_{\mathrm{f}}-k\right) \cos \theta$. The average protruding length of fiber for this sector would be $l_{2}^{*}$ given in the appendix.

$2 L_{\mathrm{c}} \leq L_{\mathrm{f}} \leq L_{2}$ : In this sector all fibers have $L_{\mathrm{f}}>2 L_{\mathrm{d}}$ and so have a potential to rupture. So, for those fibers with $l>L_{\mathrm{d}}$ the protruding length is taken as zero and for those with $l<L_{\mathrm{d}}$ the protruding length is taken as $\left(L_{\mathrm{i}}-k\right) \cos \theta$. The average protruding length of fiber for this sector would be $l_{3}^{*}$ given in the appendix.

Combining all three sectors of fibers, the average protruding length $l^{*}$ is:

$$
\begin{aligned}
l^{*}= & \int_{L_{-1}}^{2 L_{r}} l_{1}^{*} p\left(L_{\mathrm{f}}\right) \mathrm{d} L_{\mathrm{f}} \\
& +\int_{2 l_{r}}^{2 L_{*}} l_{2}^{*} p\left(L_{\mathrm{f}}\right) \mathrm{d} L_{\mathrm{f}} \\
& +\int_{2 L_{\mathrm{e}}}^{L_{2}} l_{3}^{*} p\left(L_{\mathrm{f}}\right) \mathrm{d} L_{\mathrm{f}}
\end{aligned}
$$

This equation can be solved by numerical integration using eqns (A4)-(A6) and the appropriate value of $p\left(L_{\mathrm{f}}\right)$. Figure 4 shows the plot between $l^{*}$ and $L_{\mathrm{c}}$ generated from eqn (5) for different values of $f$. The other parameters are $L_{1}=0.1 \mathrm{~mm}, L_{2}=8.1 \mathrm{~mm}, p\left(L_{\mathrm{f}}\right)=0.25$ $0.031 L_{\mathrm{f}}$ (based on actual measurement of a particular carbon fiber). This plot also coincides with the physical expectations explained for the case of composite reinforced with fibers of constant $L_{\mathrm{f}}$. Compared to Fig. 3 it can be seen from this figure that for the same values of $L_{\mathrm{c}}$ and $f$, the $l^{*}$ is much lower. This makes sense physically since in the variable $L_{\mathrm{f}}$ distribution case there are many short fibers which contribute to the low measured value of $l^{*}$. For a composite specimen reinforced with that particular fiber a $L_{\mathrm{c}}$ vs $l^{*}$ figure is created. From the experimentally measured value of $l^{*}$ and for appropriate $f$ value (for carbon fibers $f=0.5$ is assumed) the corresponding $L_{\mathrm{c}}$ is obtained. From this value of $L_{c}, \tau$ can be calculated using eqn (1).

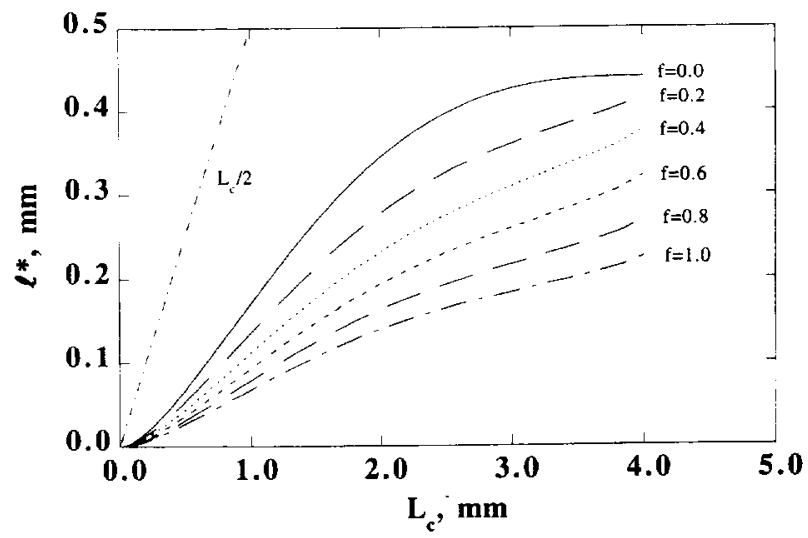

Fig. 4. Generic bond strength determination chart for fibers that display $L_{\mathrm{f}}$ distribution. 


\section{EXPERIMENTAL PROGRAM}

The objective of this experimental work is to measure the average fiber protruding length on tensile specimen fracture surfaces of the different composites using an Environmental Scanning Electron Microscope (ESEM). The carbon fiber-cement bond strength can then be estimated from the measured value of average fiber protruding length using the developed expression (eqn (5)). Uniaxial tensile testing, fiber length distribution studies and fracture surface studies were also conducted on all five sets of composites. Detailed results of fiber length distribution and tensile strength studies are described elsewhere. ${ }^{3}$ The probability density function of the fiber length after mixing is used in the $l^{*}-L_{\mathrm{c}}$ expression.

\subsection{Materials used}

The matrix used is a typical low w/c ratio cement paste matrix (see Table 1 for details). An Omni mixer of 10 liter capacity has been used for mixing the carbon fiber cement composite as it has shown to display good mixing." Five different fiber types have been used. The fiber length $L_{\mathrm{f}}$, diameter $d_{\mathrm{f}}$, tensile strength $\sigma_{\mathrm{fu}}$, Young's modulus $E_{\mathrm{f}}$, tensile strain capacity $\varepsilon_{\mathrm{fu}}$ and density $\rho$ based on manufacturer's literature are summarized in Table 2. The fiber/matrix interface bond strength $\tau$ and mean fiber length after mixing $L_{f}^{\mathrm{am}}$ are determined in this experimental program. The uniaxial tensile test results ${ }^{3}$ are summarized in Fig. 5.

\subsection{Tensile specimen fracture surface study}

Part of the tested uniaxial tensile specimen with an exposed fracture surface was placed inside

Table 1. Mix proportions

\begin{tabular}{cccc}
\hline $\begin{array}{c}\text { ASTM type III } \\
\text { cement }\end{array}$ & $\begin{array}{c}\text { Condensed } \\
\text { silica fume }\end{array}$ & $\begin{array}{c}\text { Super-plasticizer } \\
\text { (Melment) }\end{array}$ & Water \\
\hline $1 \cdot 00$ & $0 \cdot 10$ & $0 \cdot 0133$ & $0 \cdot 31$ \\
\hline
\end{tabular}

the ESEM chamber. Approximately 30 digitized images at different locations of each of the fracture surfaces was taken. Using image analysis software the protruding length of approximately 300 fibers for a given tested composite specimen was measured. The average fiber protruding length was calculated and equated to $l^{*}$ and the bond strength was evaluated using eqn (5).

(1) Figure 6 shows a computerized image from the ESEM study of the tensile specimen fracture surface of a composite reinforced with fiber F-5. There are no fiber clumps present thus indicating that the fibers are well distributed.

(2) Figure 7 shows a computerized image from the ESEM study of the tensile specimen fracture surface of a composite reinforced with fiber F-2. Even though the number of fibers present on the fracture surface $\left(4 V_{\mathrm{f}} / \pi d_{\mathrm{f}}^{2}\right.$ is the number of fibers in unit cross sectional area of composite) should be 1.74 times more than that in Fig. 6 because of the smaller fiber diameter, it can be easily seen that this is not the case. In order to give the protruding length as seen in Fig. 7 the bond strength of F-2 fiber-cement should be more than $10 \mathrm{MPa}$. Since this is unlikely it is possible that F-2 fiber suffered

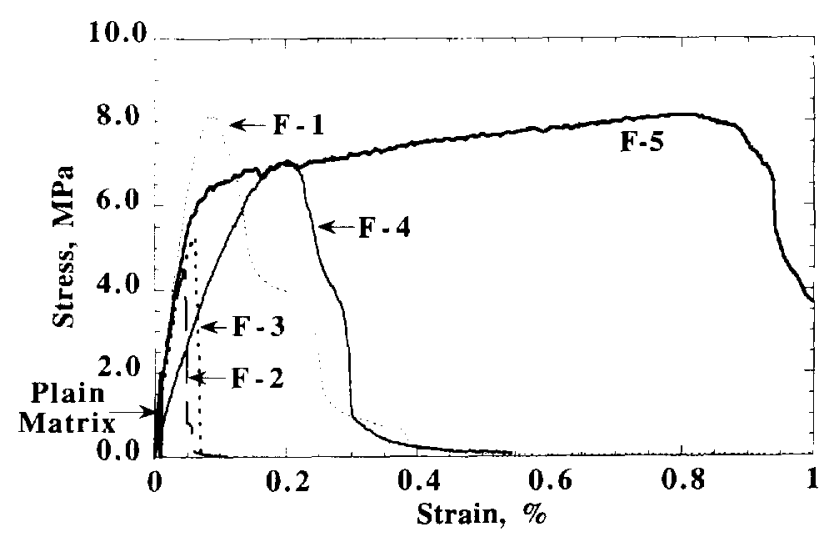

Fig. 5. Uniaxial tensile test results of cement composites reinforced with various commercially available carbon fibers.

Table 2. Fiber parameters of the different fibers used in the CFRC tensile test

\begin{tabular}{|c|c|c|c|c|c|c|c|c|}
\hline $\begin{array}{c}\text { Fiber } \\
\text { type }\end{array}$ & $\begin{array}{c}L_{\mathrm{f}} \\
m m\end{array}$ & $\begin{array}{c}d_{\mathrm{t}} \\
\mu m\end{array}$ & $\begin{array}{c}\sigma_{\mathrm{fu}} \\
M P a\end{array}$ & $\begin{array}{c}E_{1} \\
G P a\end{array}$ & itu & $\begin{array}{c}\rho \\
\mathrm{g} / \mathrm{cm}^{3}\end{array}$ & $\begin{array}{l}L_{\mathrm{f}}^{\mathrm{am}} \\
m m\end{array}$ & $\begin{array}{l}V_{\mathrm{f}} \\
\%\end{array}$ \\
\hline F-1 & 6 & $6 \cdot 8$ & 4660 & 268 & $1 \cdot 70$ & 1.77 & $2 \cdot 51$ & $3 \cdot 0$ \\
\hline $\mathrm{F}-2$ & 6 & 12 & 690 & 48 & 1.44 & 1.57 & $1 \cdot 81$ & $2 \cdot 5$ \\
\hline F-3 & 12 & 12 & 690 & 48 & 1.44 & 1.57 & $2 \cdot 58$ & $2 \cdot 5$ \\
\hline $\mathrm{F}-4$ & 3 & 24 & 800 & 38 & $2 \cdot 1$ & 1.62 & $2 \cdot 40$ & $4 \cdot 0$ \\
\hline F-5 & 10 & 20 & 800 & 38 & $2 \cdot 1$ & $1 \cdot 62$ & 6.67 & 4.0 \\
\hline
\end{tabular}




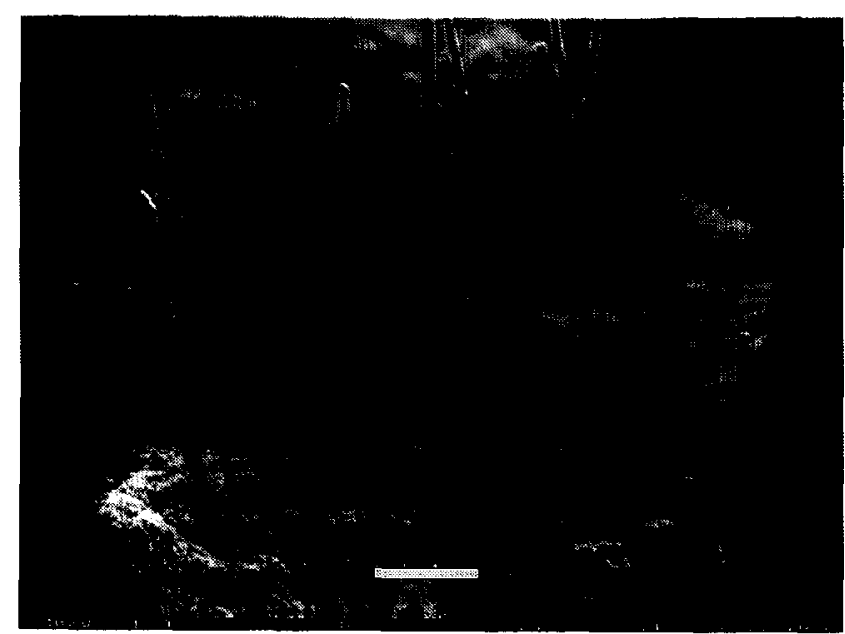

Fig. 6. ESEM of fracture surface of CFRC showing F-5 protruding fibers.

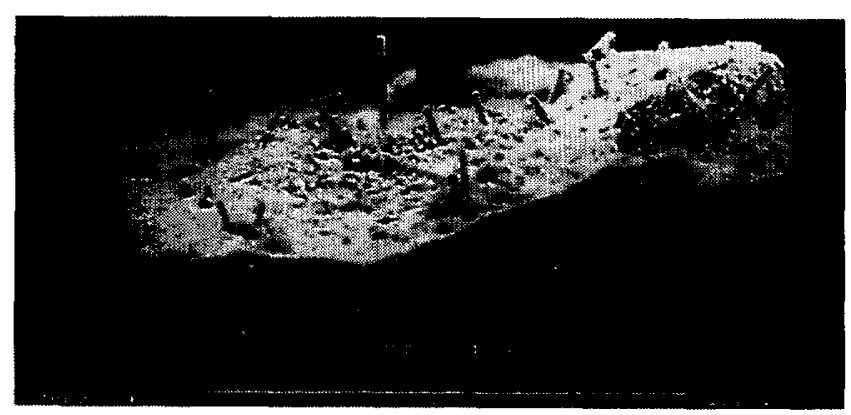

Fig. 7. ESEM of fracture surface of CFRC showing F-2 protruding fibers.

from bending rupture and so the composite did not show any pseudo strain hardening. If many of the fibers failed by bending rupture instead of tensile rupture, then the protruding lengths should be of the order of $3 d_{\mathrm{f}}$ (about $0.05 \mathrm{~mm}$ ) as the maximum stress (tensile + bending) occurs at that point. Since Fig. 6 shows that the measured average protruding length of F-5 fibers is $0.3 \mathrm{~mm}$, it can be said that fiber bending rupture is not a severe problem for F-5 fibers.

(3) The technique of measuring protruding fiber lengths based on the tensile specimen fracture surface by the ESEM has been found to be accurate. This is confirmed by the low variation of the average protruding length (only $7 \%$ ) measured between different batches.

\section{BOND PROPERTY DETERMINATION AND IMPLICATIONS OF TECHNIQUE}

The fracture surfaces of the composites prepared with the five different carbon fibers were studied. The fracture surface of the specimen

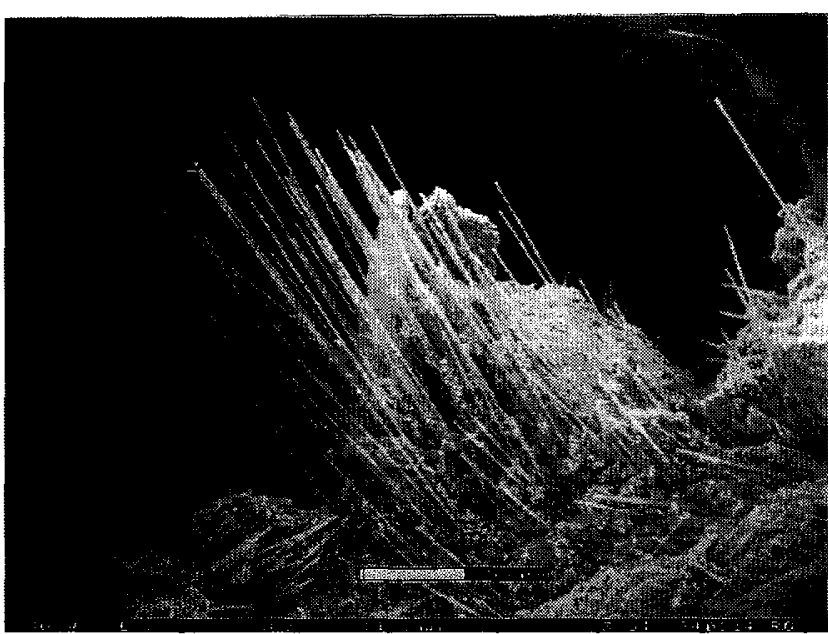

Fig. 8. ESEM of fracture surface of CFRC showing F-1 protruding fibers.

reinforced with $\mathrm{F}-1$ fibers showed a lot of fiber clumps (Fig. 8). For this technique to be applicable the fibers should not have clumps such that their whole surface area is available for bonding with cement. Any value of bond strength estimated from such a picture using the technique developed here would be inaccurate. The fracture surface study of the F-2 fiber composite shows that the average protruding length is of the order of $3 d_{\mathrm{f}}$ suggesting that most of the fibers might have failed by bending rupture. For such a case the fiber-cement bond estimation using the technique developed here would be inaccurate.

The fracture surfaces of specimens reinforced with fibers F-4 and F-5 present no such problems (Fig. 6). Two fracture surfaces of composite reinforced with fiber F-4 were studied and the measured average protruding lengths were 0.295 and $0.34 \mathrm{~mm}$. The $p\left(L_{\mathrm{f}}\right)$ has been measured ${ }^{3}$ and the corresponding $l^{*}$ vs $L_{\mathrm{c}}$ relation was drawn for $f=0.5$ (Fig. 9). $\tau$ can be estimated to be 3.10 and $2.78 \mathrm{MPa}$ for the two specimens. The higher bond strength value is for the specimen that was cured in water and the lower bond strength value is for the specimen cured in air. Using the typical fiber parameters (Table 2) the composite tensile strength has been predicted for the two specimens using an analytical model developed. ${ }^{3}$ Table 3 shows that the experimentally measured composite tensile strengths for the two specimens compare well with model predictions thus indicating that the $\tau$ predictions are accurate. Similarly two fracture surfaces of composite reinforced with fiber F-5 were studied and the 


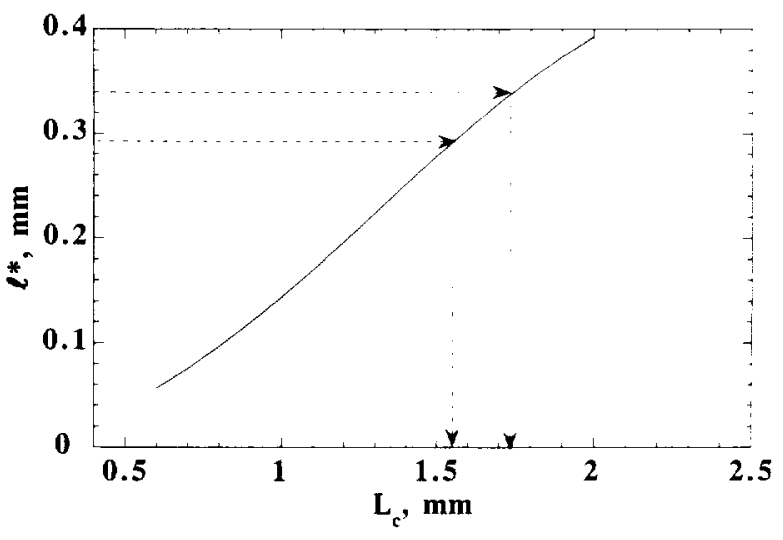

Fig. 9. Bond strength determination between F-4 fiber and cement for $f=0.5$.

Table 3. Comparisons of tensile strength predictions from analytical models with experimental results

\begin{tabular}{lllcc}
\hline Fiber & $l^{*}$ & $\tau$ & $\begin{array}{c}\text { Model } \\
\text { prediction } \\
\sigma_{\mathrm{cu}} \\
M P a\end{array}$ & $\begin{array}{c}\text { Experimental } \\
\sigma_{\mathrm{cu}}, \\
M P a\end{array}$ \\
\hline F-4 & $0 \cdot 295$ & $3 \cdot 10$ & $7 \cdot 19$ & $7 \cdot 10$ \\
& 0.340 & $2 \cdot 78$ & $6 \cdot 80$ & $5 \cdot 40$ \\
F-5 & 0.340 & $2 \cdot 06$ & $8 \cdot 80$ & $8 \cdot 20$ \\
& 0.475 & 1.76 & $8 \cdot 14$ & $6 \cdot 20$ \\
\hline
\end{tabular}

measured average protruding lengths are 0.34 and $0.475 \mathrm{~mm}$. The $p\left(L_{\mathrm{f}}\right)$ has been measured ${ }^{3}$ and the corresponding $l^{*}$ vs $L_{\mathrm{c}}$ relation was drawn for $f=0.5$ (Fig. 10). $\tau$ can be estimated to be 2.06 and $1.76 \mathrm{MPa}$ for the two specimens. The higher bond strength value is for the specimen that was cured in water and the lower bond strength value is for the specimen cured in air. Table 3 shows that the experimentally measured composite tensile strengths for the two specimens compare well with model predictions thus indicating that the $\tau$ predictions are accurate. Although all the other fiber and matrix parameters were the same the F-4 fibers had a larger diameter ( $24 \mu \mathrm{m}$ vs $20 \mu \mathrm{m})$ and a shorter length ( $3 \mathrm{~mm}$ vs $10 \mathrm{~mm}$ ) compared to F-5 fibers. Due to this, the composite reinforced by F-4 fibers had a much better workability than the composite containing F-5 fibers. This might have led to much better matrix packing around the F-4 fiber thus contributing to the higher bond strength.

Previously as there was no experimental evidence it was generally assumed that the carbon fiber cement bond strength was very high (in excess of $5 \mathrm{MPa}$ ) and to design better composites $\tau$ needed to be reduced to make the fibers

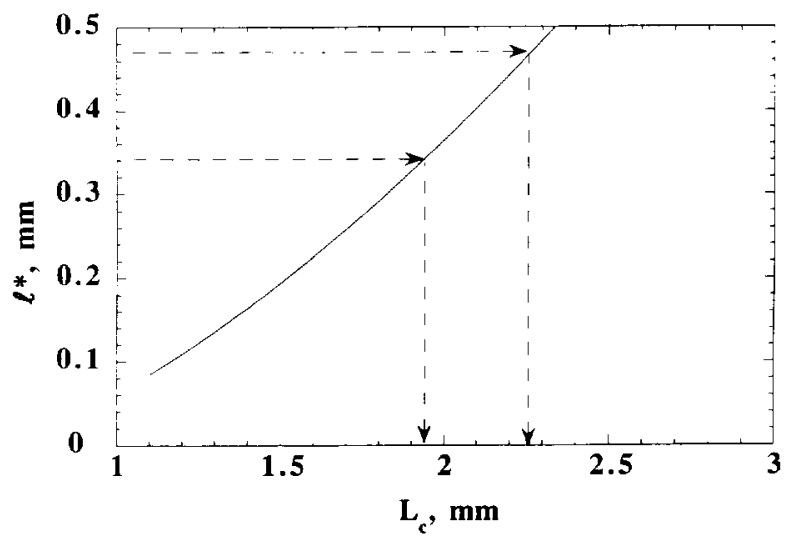

Fig. 10. Bond strength determination between F-5 fiber and cement for $f=0 \cdot 5$.

pullout instead of undergoing tensile rupture. However the results of Table 3 show that the $\tau$ for carbon fiber, at least for the ones in this study, could be as low as $1.76 \mathrm{MPa}$ and that the poor composite properties are due to other reasons such as fiber breakage during mixing. ${ }^{3}$ Another interesting finding based on the F-4 fiber reinforced cement composite results is that a $\tau$ of $3.1 \mathrm{MPa}$ is achievable in carbon fiber.

With the micro-mechanical mode ${ }^{10}$ relating composite properties to fiber, matrix and interface properties, a desirable $\tau$ can be targeted. The present bond property inference technique may be used to aid in assessing the effectiveness of various interface property modification methodology.

\section{SUMMARY AND CONCLUSIONS}

(1) The fracture surfaces of composites prepared with five different carbon fiber types were studied under an ESEM. It was found that F-1 fibers tend to clump with each other. F-2 fibers were suspected to fail by bending rupture. F-4 and F-5 fibers appeared to be uniformly distributed. The protruding lengths from the fracture surface of fibers F-4 and F-5 were measured with image analysis software and the average protruding length was of the order of $0.3 \mathrm{~mm}$ for the $\mathrm{F}-4$ fibers and $0 \cdot 34-0.475 \mathrm{~mm}$ for the F-5 fibers. Also bending rupture does not appear to be a problem for F-4 and F-5 fibers.

(2) An analytic expression has been developed which relates the 2-D measured 
average fiber protruding length from the fracture surface to the fiber-cement bond strength. The expression takes into account conversion of 2-D measured length to the actual fiber length, fiber length distribution, fiber embedment length distribution, and snubbing effect. A similar analytic expression relating the 2-D measured length to $\tau$ has been developed for the case of constant fiber length. This is useful for fibers that break but do not have a length distribution like carbon fibers (e.g. polypropylene fibers).

(3) Using the developed technique, bond strengths of $2 \cdot 78-3 \cdot 10 \mathrm{MPa}$ and 1.76-2.06 $\mathrm{MPa}$ were predicted for $\mathrm{F}-4$ and F-5 fibers, respectively. Using these $\tau$ values composite tensile strengths were predicted for four different specimens and the correlation with experimental results of uniaxial tensile tests proved to be accurate. This indicates that the bond strength estimates were correct thus ensuring the validity of the technique developed. The bond strength appears to be affected by the fiber length and volume fraction which in turn affect workability.

(4) This experimental technique provides a method to measure $\tau$ for different fibers or for the same fiber with different surface treatments. Thus it serves as a tool to develop carbon fiber cement composites with optimum properties in the future. It can also be used for measuring the bond strengths of any fiber that ruptures after cracking of the brittle matrix other than cement.

\section{ACKNOWLEDGEMENTS}

This research has been supported by a contract with Conoco Inc. to the University of Michigan. The authors acknowledge helpful discussions with Drs J. McConaghy and A. Katz.

\section{REFERENCES}

1. Larson, B. K., Drzal, L. T. \& Sorousian, P., Carbon fiber-cement adhesion in carbon fibre reinforced cement composites. Composites, 21(3) (1990) 205-16.

2. Katz, A. \& Li, V. C., A special technique for determining the bond strength of carbon fibers in cement matrix by pull-out test. J. Mater. Sci. Lett. (submitted).

3. Li, V. C. \& Obla, K. H., Effect of fiber length variation on tensile properties of carbon fiber cement composites. Int. J. Compos. Engng, 4(9) (1994) 947-64.

4. Kelly, A. \& Macmillan, N. H., Strong Solids, 3rd edn. Clarendon Press, Oxford, 1986.

5. Li, V. C., Wang, Y. \& Backer, S., Effect of inclining angle, bundling, and surface treatment on synthetic fiber pull-out from a cement matrix. J. Compos., 21(2) (1990) 132-40.

6. Nishioka, K., Yamakawa, S. \& Shirakawa, K., Properties and applications of carbon fibre reinforced cement composites. Proc. RILEM Symp., 1986.

7. Linton, J. R., Berneburg, P. L., Gartner, E. M. \& Bentur. A., Carbon fiber reinforced cement and mortar. Mater. Res. Soc. Symp. Proc., Vol. 211, pp. 255-64. Materials Research Society, 1991.

8. Akihama, S., Kobayashi, M., Suenaga, T., Nakagawa, H. \& Suzuki, K., Mechanical properties of carbon fiber reinforced cement composite and the application to buildings. KICT Rep., No. 65 (1986) Kajima Corporation, Tokyo-182, Japan.

9. Banthia, N., Pitch-based carbon fiber reinforced cements: structure, performance, applications, and research needs. Can. J. Civil Engng, 19 (1992) 26-38.

10. Li, V. C., From micro-mechanics to structural engineering - the design of cementitious composites for civil engineering applications. J. Structural Mech. Earthquake Engng, JSCE, 10(2) (1993) 37-48.

\section{APPENDIX}

\section{Derivation of $l^{*}$ vs $L_{\mathrm{c}}$ relation}

The average protruding length for the different sectors can be written as:

$$
\begin{aligned}
l_{1}^{*}= & 2 M\left(0, \pi / 2-\phi, \phi, L_{\mathrm{f}} / 2, L_{\mathrm{f}}\right) \\
& \text { for } 2 L_{1} \leq L_{\mathrm{f}} \leq 2 L_{\mathrm{r}} \\
l_{2}^{*}= & 2\left[M\left(\phi_{1}, \pi / 2,-\phi, \phi, L_{\mathrm{f} 1}, L_{\mathrm{f}}\right)\right. \\
& \left.+M\left(0, \phi_{1},-\phi, \phi, L_{\mathrm{f}} / 2, L_{\mathrm{f}}\right)\right] \\
& \text { for } 2 L_{\mathrm{r}} \leq L_{\mathrm{f}} \leq 2 L_{\mathrm{c}} \\
l_{3}^{*}= & 2\left[M\left(0, \pi / 2,-\phi, \phi, L_{\mathrm{f} 1}, L_{\mathrm{f}}\right)\right] \\
& \text { for } 2 L_{\mathrm{c}} \leq L_{\mathrm{f}} \leq L_{2}
\end{aligned}
$$

where

$$
\begin{gathered}
M(a, b, c, d, e, f) \\
=\int_{a}^{b} \int_{c}^{d} \int_{e}^{f}\left(\frac{1}{L_{\mathrm{f}}}\right)\left(\frac{1}{2 \phi}\right) \\
\quad \times\left(L_{\mathrm{f}}-k\right) \cos \theta \sin \phi \mathrm{d} k \mathrm{~d} \theta \mathrm{d} \phi \\
L_{\mathrm{f} 1}=L_{\mathrm{f}}-L_{\mathrm{c}} \mathrm{e}^{-f \phi}, \phi_{1}=-\frac{1}{f} \ln \left(\frac{L_{\mathrm{f}}}{2 L_{\mathrm{c}}}\right), \\
L_{\mathrm{r}}=L_{\mathrm{c}} \mathrm{e}^{-f \pi / 2}, L_{\mathrm{d}}=L_{\mathrm{c}} \mathrm{e}^{-f \phi}
\end{gathered}
$$


After simplifications,

$$
l_{1}^{*}=0 \cdot 2059 L_{\mathrm{f}}
$$

$l_{2}^{*}+\int_{\phi_{1}}^{\pi / 2} \frac{L_{\mathrm{c}}^{2}}{L_{\mathrm{f}} \phi} \mathrm{e}^{-2 f \phi}(\sin \phi)^{2}+\int_{0}^{\phi_{1}} \frac{L_{\mathrm{f}}}{4 \phi}(\sin \phi)^{2} \mathrm{~d} \phi$

$$
l_{3}^{*}=\int_{0}^{\pi / 2} \frac{L_{\mathrm{c}}^{2}}{L_{\mathrm{f}} \delta} \mathrm{e}^{-2 f \phi}(\sin \phi)^{2} \mathrm{~d} \phi
$$

The average fiber length protruding from the tensile specimen fracture surface $l^{*}$ would be given by eqn (5)

(A5) 